\title{
Allele-specific silencing of EEC p63 mutant R304W restores p63 transcriptional activity
}

\author{
F Novelli ${ }^{1}$, AM Lena ${ }^{1}$, E Panatta ${ }^{1}$, W Nasser ${ }^{2}$, R Shalom-Feuerstein ${ }^{2}$, E Candi ${ }^{1}$ and G Melino*,1,3
}

EEC (ectrodactily-ectodermal dysplasia and cleft lip/palate) syndrome is a rare genetic disease, autosomal dominant inherited. It is part of the ectodermal dysplasia disorders caused by heterozygous mutations in TP63 gene. EEC patients present limb malformations, orofacial clefting, skin and skin's appendages defects, ocular abnormalities. The transcription factor p63, encoded by TP63, is a master gene for the commitment of ectodermal-derived tissues, being expressed in the apical ectodermal ridge is critical for vertebrate limb formation and, at a later stage, for skin and skin's appendages development. The $\Delta N p 63 \alpha$ isoform is predominantly expressed in epithelial cells and it is indispensable for preserving the self-renewal capacity of adult stem cells and to engage specific epithelial differentiation programs. Small interfering RNA (siRNA) offers a potential therapy approach for EEC patients by selectively silencing the mutant allele. Here, using a systemic screening based on a dual-luciferase reported gene assay, we have successfully identified specific siRNAs for repressing the EEC-causing p63 mutant, R304W. Upon siRNA treatment, we were able to restore $\triangle$ Np63-WT allele transcriptional function in induced pluripotent stem cells that were derived from EEC patient biopsy. This study demonstrates that siRNAs approach is promising and, may pave the way for curing/delaying major symptoms, such as cornea degeneration and skin erosions in young EEC patients.

Cell Death and Disease (2016) 7, e2227; doi:10.1038/cddis.2016.118; published online 19 May 2016

EEC syndrome (ectrodactily-ectodermal dysplasia and cleft lip/palate syndrome, OMIM\#604292) is an autosomal dominant rare disorder characterized by abnormal development of tissues that originate from ectoderm. Clinical features include: limbs malformations, orofacial clefting, skin, skin's appendage and ocular defects. ${ }^{1-4}$ The severity and type of ectodermal defects is highly variable and they may depend on the type of mutations. ${ }^{5}$ EEC patients often present skin manifestation, such as hyperkeratosis, erythroderma, skin erosion and ocular defects because of limbal stem cell deficiency (LSCD). ${ }^{2,4}$ Limbal stem cells, located in the limbus, at the cornea periphery, are indispensable for cornea formation. EEC patients undergo to a progressive degeneration of the corneal epithelial tissue, generally reaching a peak in the third decades, leading to corneal opacity, poor vision, neovascularization and irreversible blindness. ${ }^{3,6-8}$

The transcription factor p63, encoded by TP63 gene, part of the p53 gene family, is a master gene for the commitment of ectodermal-derived tissues. The action of p63 is likely to be required for skin development and for the formation of the apical ectodermal ridge (AER), which has a critical role in distal outgrowth and patterning of the vertebrate limb and, subsequently, in skin and skin's appendages formation. ${ }^{6}$ Missense mutations in TP63 are the cause of at least seven rare ectodermal dysplasia (ED) disorders, including EEC. ${ }^{9,10}$ TP63 is a complex gene, generating six different proteins because of the use of two alternative promoters (P1 and P2) that give rise to the $\mathrm{N}$-terminal truncated and full-length isoforms, respectively, $\Delta \mathrm{Np} 63$ and TAp63, and alternative $\mathrm{C}$-terminus splicing, generating $\alpha, \beta$ and $\gamma$ isoforms. The $\Delta \mathrm{Np63a}$ is the predominant isoform expressed in epithelial cells, in different epithelial tissues, including, epidermis, thymic epithelial cells, cornea, prostate, mammary gland, it is indispensable for preserving the self-renewal capacity of adult stem cells. ${ }^{11-14}$ This is also confirmed by the $\Delta$ Np63 isoform-specific knock-out mice phenotype, ${ }^{15}$ which nicely recapitulate human ED symptoms. Besides stemness, p63 is also responsible for regulating different cellular pathways, including cellular metabolism and anti-oxidant response, cellular adhesiveness and cytoskeleton, epithelial differentiation program, cellular migration. ${ }^{16-21}$

The current therapy for disorders caused by p63 mutations is limited to surgery and is supportive and not curative. Interestingly, five p63 DNA-binding mutations account for almost $90 \%$ of EEC patient cases. The five missense mutations affect arginine residues (R204, R227, R279, R280 and R304) and represent hotspot mutations for EEC. ${ }^{1}$ These mutants, similarly to other p63 mutants, are translated into proteins with enhanced stability, ${ }^{22}$ maintain the ability to bind DNA but, being transcriptionally deficient themselves and by inhibiting transactivation by the residual p63 wild-type allele, have a strong dominant-negative effect., ${ }^{1,9,22}$ The lack of therapies and the presence of hotspot mutants in EEC patients, lead us to explore the possibility that using

\footnotetext{
${ }^{1}$ Department of Experimental Medicine and Surgery, University of Rome 'Tor Vergata', Rome, Italy; ${ }^{2}$ Department of Genetics and Developmental Biology, The Ruth and Bruce Rappaport Faculty of Medicine, Technion-Israel Institute of Technology, Haifa, Israel and ${ }^{3}$ Medical Research Council, Toxicology Unit, Leicester University, Hodgkin Building, Leicester, UK

${ }^{*}$ Corresponding author: G Melino, Department of Experimental Medicine and Surgery, University of Rome 'Tor Vergata', via Montpellier, Rome 100133, Italy. Tel: +39 6204 27 299; Fax: +39 620427 290; E-mail: gm89@leicester.ac.uk or gerry.melino@ uniroma2.it

Abbreviations: EEC, ectrodactily-ectodermal dysplasia and cleft lip/palate; ED, ectodermal dysplasia; AER, apical ectodermal ridge; LSCD, limbal stem cell deficiency; siRNA, small interfering RNA; DBD, DNA-binding domain

Received 26.8.15; revised 03.3.16; accepted 08.3.16; Edited by A Stephanou
} 
allele-specific gene silencing it could selectively inhibit the expression of the disease-associated allele without suppressing the expression of the wild-type allele, therefore restoring the single functional p63 allele, still sufficient for healthy tissue development and maintenance. Here, we designed and selected effective small interfering RNAs (siRNAs) molecules for $\triangle$ Np63-R304W EEC mutant using transfected cell line, and examined the efficiency of allele-specific knockdown. We

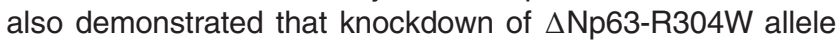
restore $\Delta \mathrm{Np63-WT}$ allele transcriptional activity. These results

a

$\triangle$ Np63a-WT

5' -TCCTGGGCCGACGCTGCTTTGAGGCCCGGATCTGTGCTTGCCCAGGAAGA-3'

$\triangle \mathrm{Np63 \alpha -R304W}$

5' -TCCTGGGCCGACGCTGCTTTGAGGCC ThGATCTGTGCTTGCCCAGGAAGA-3'

b

\begin{tabular}{|c|c|}
\hline NA-T19 & CGACGCUGCUUUGAGGCCUUU \\
\hline siRNA-T18 & GACGCUGCUUUGAGGCCUGUU \\
\hline siRNA-T17 & ACGCUGCUUUGAGGCCUGGGU \\
\hline siRNA-T16 & 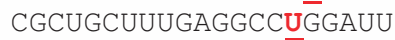 \\
\hline siRNA-T15 & GCUGCUUUGAGGCCUGGGA \\
\hline siRNA-T14 & CUGCUUUGAGGCCŪḠGAU \\
\hline siRNA-T13 & UGCUUUGAGGCCŪGGAUC \\
\hline siRNA-T12 & 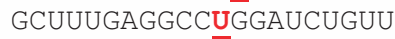 \\
\hline siRNA-T11 & CUUUGAGGCCŪḠGAUCUGUL \\
\hline siRNA-T10 & UUUGAGGCCUḠGAUCL \\
\hline siRNA-T9 & UUGAGGCCŪGGAUCUGUGCL \\
\hline siRNA-T8 & UGAGGCCŪḠGAUCUGUGCL \\
\hline siRNA-T7 & GAGGCCUGGAUCUGUGCUUU \\
\hline siRNA-T6 & AGGCCUGGGUCUGUGCUUGL \\
\hline siRNA-T5 & GGCCUGGGAUCUGUGCUUG \\
\hline siRNA-T4 & GCCUGGAUCUGUGCUUGCCL \\
\hline siRNA-T3 & CCUGGGAUCUGUGCL \\
\hline siRNA-T2 & CUGGGAUCUGUGCUL \\
\hline siRNA-T1 & GCL \\
\hline
\end{tabular}

C
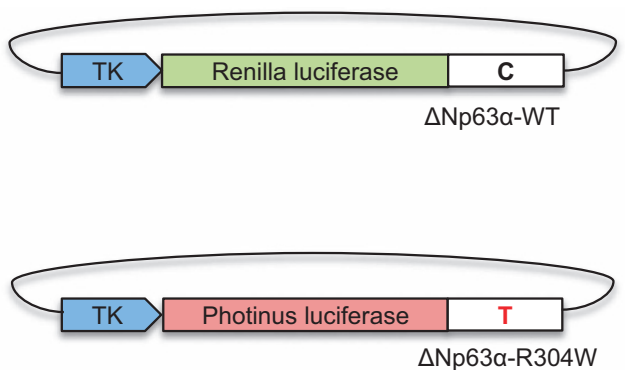

d
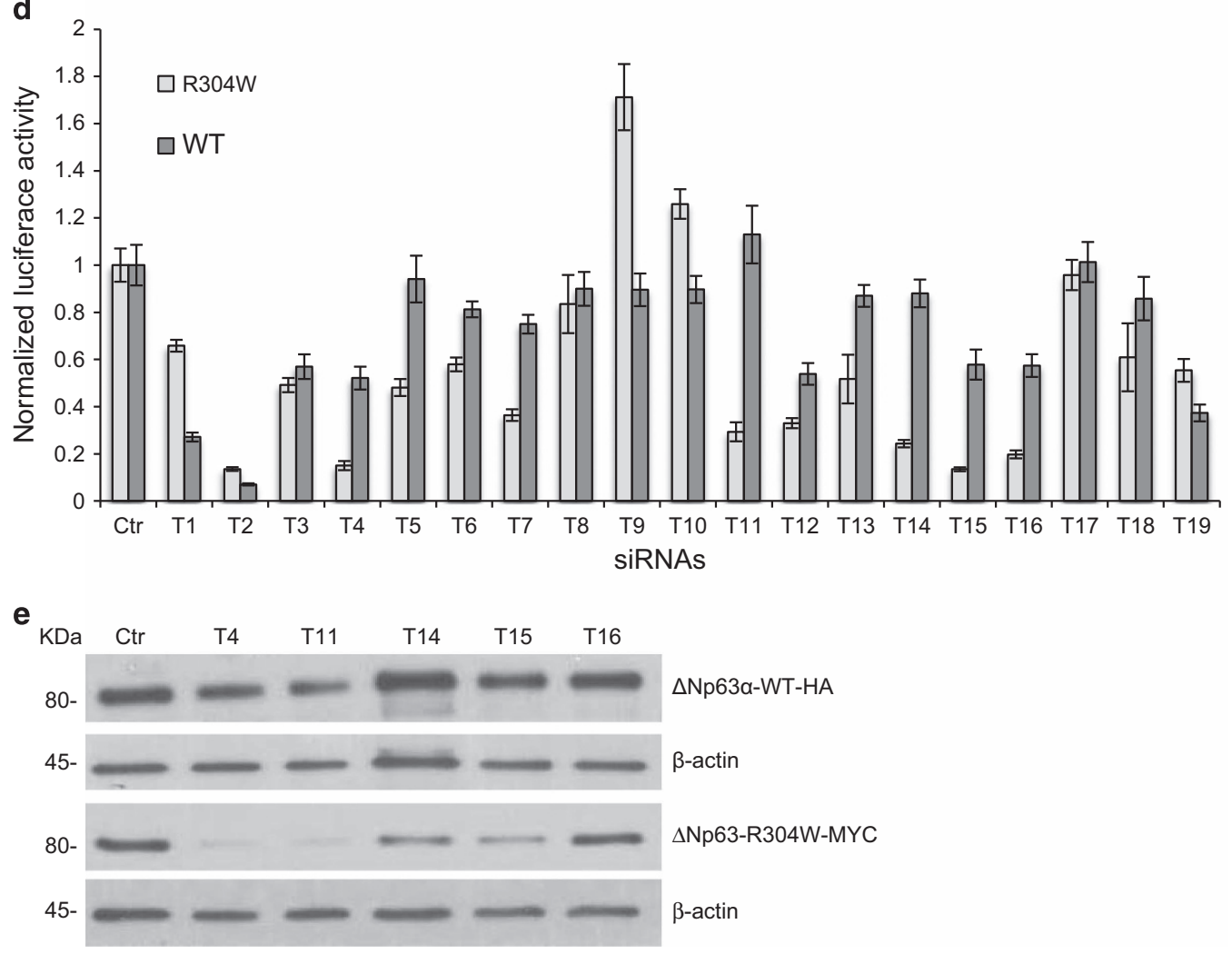
indicate that allele-specific silencing of the mutant mRNA can potentially be considered as a therapeutic procedure in EEC, and, more in general, ED patients because of p63 point mutations. This strategy could be relevant to cure/prevent/ delay selected clinical features such as skin erosion and loss of corneal function in younger patients.

\section{Results}

Design and selection of optimal siRNA molecules. A sequence walk was performed whereby all 19 possible siRNA molecules were designed to span and include $\Delta$ Np63R304W point mutation, as shown in Figure 1. For screening of siRNA molecules blocking $\triangle$ Np63-R304W expression with little or no effect on $\Delta$ Np63-WT, we used two kinds of artificial report constructs ${ }^{23}$ harboring Renilla luciferase or Photinus luciferase and 50-bp siRNA-targeted sequences with/without $\mathrm{C}>\mathrm{T}$ substitution in their 3'-UTRs (Figures 1a-c). Each siRNA molecule was investigated for its ability to suppress

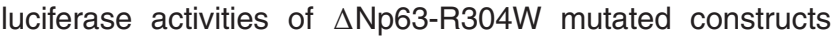
significantly compared with those of $\triangle \mathrm{Np63-WT}$ ones by transient transfection in HEK-293E epithelial cells.

Preferential inhibition of $\Delta$ Np63-R304W versus $\Delta$ Np63-WT by specific siRNAs. In order to identify mutated allelespecific siRNAs, we performed luciferase assay upon HEK-293E transient transfection using the reported vector described in Figures 1a-c. Results indicated that almost all siRNAs tested suppressed the luciferase activities of mutated and WT constructs. Some of them were not discriminatory, inhibiting both $\Delta$ Np63-WT and $\Delta$ Np63-R304W mutant (T1, T2, T8 and T12). Some siRNAs tested had little or no effect on luciferase activity of either $\Delta$ Np63-WT or $\Delta$ Np63R304W mutant reporter constructs (T9, T10 and T17). From the 19 siRNAs tested, 10 molecules, T3, T4, T5, T6, T7, T11, T14, T15, T16 and T18, were able to specifically suppress luciferase activity of the mutant allele-containing reporter construct with little effects on the one bearing the WT allele (Figure 1d). Although all of these had good potential for therapeutic use, we further validated them focusing on those suppressing luciferase activity to a maximum of $40 \%$ for $\Delta$ Np63-WT allele and at least $80 \%$ for $\Delta$ Np63-R304W mutant allele (T4, T11, T14, T15 and T16). We then analyzed the gene suppression efficiencies of the selected siRNAs on WT/R304W full-length transcripts. HEK-293E cells were transfected with HA-tagged wild-type (pcDNA-HA- $\triangle$ Np63) and Myc-tagged mutant (pcDNA-Myc- $\triangle$ Np63-R304W) constructs, as an in vitro cellular model of the EEC diseaserelated heterozygous status, and the five representative discriminatory siRNAs (T4, T11, T14, T15 and T16) at a concentration of $40 \mathrm{nM}$. The data show that siRNAs T4 and $\mathrm{T} 11$ are highly potent and highly specific for the p63 mutation $\mathrm{R} 304 \mathrm{~W}$ at the level of protein expression (Figure 1e), whereas T14, T15 and T16 were less efficient to knockdown $\Delta$ Np63R304W mutant at protein level. This experiment indicates that the siRNAs identified, especially $\mathrm{T} 4$ and $\mathrm{T} 11$, are selective for the mutated allele, strongly knocking-down the $\Delta$ Np63-R304W mutant transcript, resulting in mutated protein translation inhibition.

Silencing of $\Delta \mathrm{Np63-R304W}$ mutant partially rescues $\Delta \mathrm{Np63a-WT}$ transcriptional activity. Having demonstrated the effectiveness and the selectivity of the siRNAs identified for $\Delta$ Np63-R304W mutant allele, we checked whether these (T4 and T11) could restore the transcriptional activity of $\triangle$ Np63-WT. As experimental system, we used HEK-293E cells monitoring the endogenous $\Delta$ Np63-driven targets keratin 14 (K14) and p53 effector related to PMP-22 (PERP) mRNAs expression. HEK-293E cells were transiently transfected with $\Delta$ Np63-WT, $\Delta$ Np63-R304W and $\Delta$ Np63-WT: $\triangle$ Np63-R304W (2:1 ratio) in presence of not specific siRNA (Scr), T4 or T11. K14 mRNA increases 68-fold after $\Delta$ Np63WT transient transfection, whereas its transcriptional activity

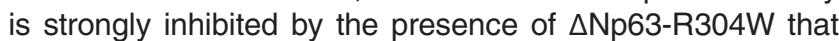
has a well-known dominant-negative effect on $\Delta$ Np63-WT transcriptional activity, also at 2:1 ratio (Figure 2a). Interestingly, addition of $40 \mathrm{nM} \mathrm{T4}$ or T11 partially rescued $\Delta$ Np63WT function, increasing K14 mRNA to 2.2- and 2.8-fold,

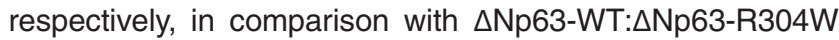
transfected cells with not specific siRNA (Scr) (Figure 2a). Similar results were obtained for PERP mRNA. Indeed, addition of $40 \mathrm{nM}$ T4 or T11 partially rescued $\triangle$ Np63-WT function, increasing PERP mRNA to 1.8- and 2.2-fold,

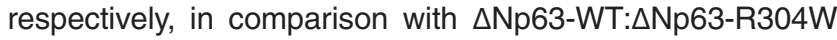
transfected cells with not specific siRNA (Scr) (Figure 2b). Western blot confirmed T4 and T11 efficacy of selective silencing (Figure 2c). These results were also confirmed in Saos-2 cell line. $\Delta$ Np63-WT, $\Delta$ Np63-R304W and $\Delta$ Np63-WT: $\Delta$ Np63-R304W (2:1 ratio) in presence of not specific siRNA (Scr), T4 or T11, were transiently transfected in Saos-2 cells and obtained partial or total rescue of K14, PERP and EVPL

\footnotetext{
Figure 1 A comprehensive siRNA walk for p63-R304W mutant silencing. (a) Nucleotide sequence of WT and mutant p63 transcript. WT and mutated sequences around mutation site (in bold) are shown. (b) In order to screen all possible sequences containing the R304W mutation, 19 siRNAs were designed, each of which consisting of 19 nucleotides with two uracil nucleotides overhangs. (c) Schematic drawing of reporter alleles. Reporter alleles were constructed based on Photinus and Renilla luciferase reporters driven by TK promoter. WT, Renilla luciferase gene with sequence of WT p63 in 3'-UTR; Mutant, Photinus luciferase gene with sequence of p63-R304W in 3'-UTR. (d) Each siRNA under evaluation was co-transfected into HEK-293E cells, at $40 \mathrm{nM}$, with either a WT-p63 luciferase reporter construct ( $\mathrm{pRL}$-TK-WT) and the mutant p63 luciferase reporter construct (pGL-TK-R304W). The sequence walk shows that several siRNAs were selective for the mutant sequence. The best are T4, T11, T14, T15 and T16. Luciferase activity was normalized also for transfection efficiency by $\beta$-galactosidase activity (PSV- $\beta$ gal, co-transfection) and nonspecific siRNA was used as a negative control (Ctr). Values are calculated relative to nonspecific siRNA using average of three to six experiments. Data are presented as mean \pm S.D. (e) HEK-293E cells were transfected with HA-tagged $\Delta$ Np63-WT (pcDNA-HA- $\Delta$ Np63) and Myc-tagged $\Delta$ Np63-R304W mutant (pcDNA-Myc- $\Delta$ Np63-R304W) constructs and five representative discriminatory siRNAs (T4, T11, T14, $\mathrm{T} 15$ and T16) at $40 \mathrm{nM}$. These data show that siRNAs T4 and T11 are highly potent and highly specific for the p63 R304W mutated allele, inhibiting at high-level mutant protein expression but not $\Delta$ Np63-WT. One representative experiment of three is shown. $\beta$-Actin is shown as loading control
} 

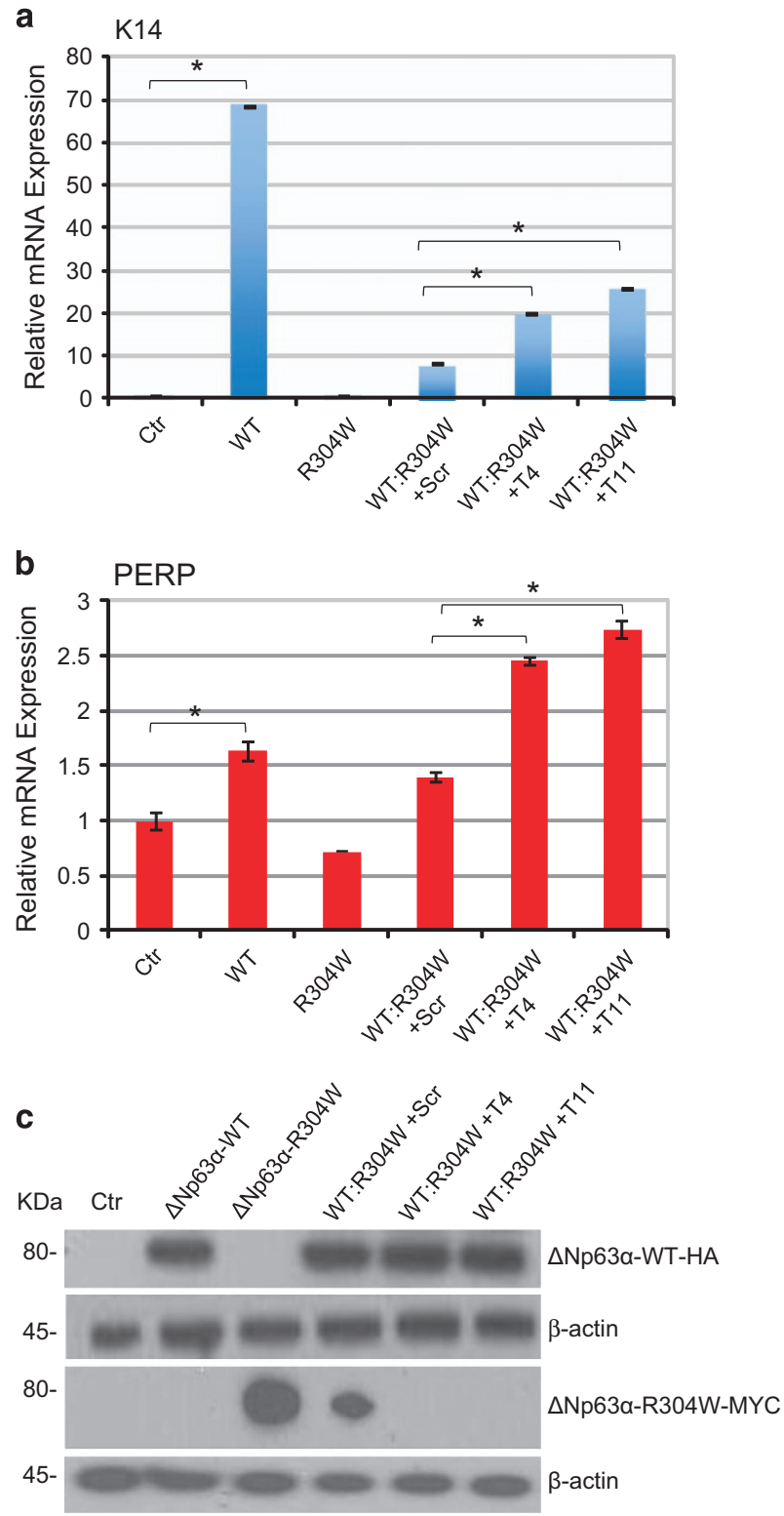

Figure 2 Silencing of the mutant allele restores $\triangle$ Np63-WT transcription activity. (a) HEK-293E cells were transfected with $\triangle$ Np63-WT, $\Delta$ Np63-R304W, $\Delta$ Np63-WT: $\Delta$ Np63-R304W (2:1 ratio) in presence of siRNA not specific sequence (Scr), T4 and T11. $\Delta$ Np63-WT is HA-tagged; $\Delta$ Np63-R304W is Myc-tagged. T4 and T11 partially restore $\Delta$ Np63-WT transcriptional activity, evaluated by real-time PCR of keratin 14 (K14) mRNA. Data are presented as mean \pm S.D. of three independent experiments. ${ }^{\star} P<0.05$. (b) Experiment was performed as described in a) T4 and T11 partially restore $\triangle$ Np63-WT transcriptional activity, evaluated by real-time PCR of PERP mRNA. Data are presented as mean \pm S.D. of three independent experiments. ${ }^{*} P<0.05$. (c) Western blot demonstrates $\mathrm{T} 4$ and $\mathrm{T} 11$ selectivity. One representative experiment of three is shown. $\beta$-Actin is shown as loading control

mRNAs (Supplementary Figures S1a and b), because of allele selective silencing by $\mathrm{T} 4$ and $\mathrm{T} 11$. These results show that selective silencing of the $\Delta$ Np63-R304W mutated allele restore $\triangle$ Np63-WT transcriptional activity, as shown by the significant increases in endogenous p63 targets mRNAs (K14, PERP and EVPL) upon siRNAs treatments.
Rescue of corneal epithelial differentiation of EEC-iPSCs by mutated-p63 allele-specific siRNAs. Based on the efficient repression of p63 mutant targeting siRNAs in HEK-293E cells, we aimed at testing the effect in a more physiologically relevant model using patients' cells. We have recently generated induced pluripotent stem cells (iPSCs) from p63-R304W-EEC patients and showed that these cells can serve as an in vitro model for testing novel therapeutic approaches. $^{7}$ In contrast to primary cultures of patient keratinocyte, iPSCs provide a durable model because they can be expanded forever in an undifferentiated state and directed to lineage-specific fates including epidermal and corneal lineages, whereas EEC patient cells have a very short life span. To test the possibility that siRNA can restore patient-specific iPSCs (EEC-iPSCs), we chose to study corneal differentiation because of the high efficiency of this differentiation model. ${ }^{24}$ Normal iPSCs (WT-iPSCs) and EEC-iPSCs were seeded on collagen IV-coated dishes in the presence of medium conditioned by human corneal fibroblasts. As illustrated in Figures $3 a$ and b, iPSC lines underwent sequential differentiation into corneal fate and displayed epithelial characteristics as evident by their morphology and gene expression. As shown in Figures 3b-d, although WT-iPSC could fully commit into epithelial lineage, EEC-iPSC displayed partial differentiation as evident by reduced expression of $\mathrm{K} 14$, and two other additional p63-target genes, GJ6B and DIx5. ${ }^{7}$ Accordingly, we used this system to test whether mutated allele-specific siRNA can restore the function of p63 mutant function and promote corneal differentiation of EEC-iPSC. For that purpose, EEC-iPSCs were subjected to differentiation and transfection with specific siRNAs (T4 and T11) or control siRNA (Scr) was performed at days $8-10$ of differentiation because at this stage cells begin to express p63 and undergo commitment from ectoderm to stratified epithelium. ${ }^{24}$ Cells were collected at days $13-15$ of differentiation and the impact of siRNA treatment on cell differentiation was determined by real-time QPCR and flow cytometry analysis of K14. Interestingly, under transfection of $\mathrm{T} 4$ or $\mathrm{T} 11$, cell differentiation was partially rescued (Figures $3 e$ and f). GJ6B, which is linked with ED and, previously shown, to be elevated in WT mice but not in p63-null mice at embryonic day $14.5,{ }^{6}$ was rescued by $\mathrm{T} 4$ and $\mathrm{T} 11$ (six and threefold, respectively). Similarly, the mRNA level of DLX5, a p63-target gene that is associated with EEC syndrome, was enhanced by T4 and T11, 27- and 13-fold, respectively. Finally, real-time PCR and flow cytometry analyses confirmed silencing efficacy of T4 and T11 with an increase in K14 mRNA (9- and 5.5-folds, respectively; Figure $3 e$ ) and in the fraction of K14-positive cells (Figure $3 \mathrm{f}$ ).

\section{Discussion}

In this study, we developed a method for allele-specific gene silencing with siRNAs that target a TP63 R304W missense mutation, found in patients affected with autosomal dominant EEC syndrome. EEC syndrome, part of ED disorders, is caused by p63 mutations localized in the DNA-binding domain (DBD). Several efforts to understand the mechanism of disease in EEC have shown that p63 DBD mutants have 
both loss their ability to transactivate target genes and also have increased protein stability, ${ }^{22}$ resulting in a strong dominant-negative effect toward the p63-WT allele. ${ }^{1,22}$ As TP63 encodes an essential protein, indispensable to maintain adult stem cells proliferative potential and to engage specific differentiation programs, treatment would necessitate to selectively inhibit the mutant allele expression without affecting WT allele. Several methods to modulate gene expression have been developed, including gene editing by zinc-finger nuclease (TALEN, CRISPR/Cas) and modification of the transcripts by exon skipping, and protein inhibitors by antibodies and drugs. ${ }^{25-27}$ Among these methods, we believe that allele-specific knockdown could be a highly relevant therapeutic method for EEC syndrome, and possibly other ED disorders, because the mutated-p63 protein form a high order complex with WT p63 protein (tetramerization), therefore showing the dominant-negative effect.

Five hotspot missense mutations, accounting for $90 \%$ of the mutations identified in EEC, have been identified, among them R304W, suggesting that by selecting few siRNA molecules one would be able to treat the majority of the EEC patients. ${ }^{1}$ We think that the siRNAs identified could be used topically to cure/delay selected clinical onsets with high impact on the patient quality of life, by attenuating skin erosion and ocular defects (Figure 4). EEC patients often have skin manifestation, some patients experience aplasia cutis congenital of the scalp and back, a debilitation clinical manifestation with no treatment. In these patients, skin defects heal very slowly and may recur periodically throughout childhood. In addition, EEC patients result in a specific ocular phenotype-involving cornea. The cornea epithelium is regenerated physiologically by p63-expressing limbal stem cells. The major case of ocular defect in these patients is LSCD, resulting in a progressive degeneration of corneal epithelial tissue, generally reaching a peak on the third and fourth decade of patients' life. When this occurs, conjuctival epithelium migrates over the cornea leading to cornea opacifiation, neovascularization, poor vision and irreversible blindness. Such slow aggravation in corneal abnormalities provides a large window for preventive intervention. Our results suggest that preventive strategies applied in early stage of the disease, could help young patients to correct/delay the EEC defects. Depending on the severity of the symptoms, siRNA molecules could be applied topically, as body cream or eye drops formulations, following specific indications (Figure 4). Similarly, a preventive strategy may be applied to counteract dermatological defects in EEC patients. The use of siRNAs in dermatological dominantnegative disorders have been explored also in other studies for keratin mutations in epidermolysis bullosa simplex ${ }^{28}$ and epidermolitic palmoplantar keratoderma. ${ }^{29}$ In ocular disease, the use of allele-specific siRNA silencing has been investigated in Meesmann epithelial corneal dystrophy ${ }^{30-32}$ and in Lattice corneal dystrophy type I. $^{33}$ Altogether, this strategy could be widely applied for treating dominantly inherited pathologies and is currently under investigation in clinical trials.

Here, we demonstrated a proof-of-principle approach to select highly potent and mutant-specific siRNA molecules that target p63 mutated allele causing EEC syndrome. The next challenge is the development of efficacious non-invasive delivery system to get these small molecules to the ocular surface and to cross skin outer layers. A similar strategy could be used also for other p63-dependent ED pathologies.

\section{Materials and Methods}

Cell culture and cells transfection. HEK-293E cells were cultured in Dulbecco's modified Eagle medium (DMEM-F12, Lonza, Basel, Switzerland) supplemented with $10 \%$ fetal bovine serum (Gibco, Life Technologies, Carlsbad, CA, USA). For transfection, HEK-293E cells were seeded at $1 \times 10^{6}$ cells into $10 \mathrm{~cm}$ Petri dishes. Cells were transfected using Lipofectamine 2000 (Invitrogen, Carlsbad, CA, USA) according to the manufacturer's instructions. HEK-293E cells were transfected with HA-tagged $\Delta$ Np63-WT (pcDNA-HA- $\Delta$ Np63 $\alpha$ ) and Myc-tagged $\Delta$ Np63-R304W mutant (pcDNA-Myc- $\Delta$ Np63 $\alpha$-R304W) constructs and R304W mutation-specific siRNAs T4 and T11 at a concentration of $40 \mathrm{nM}$. The total amount of transfected DNA was $14 \mu \mathrm{g}$ per dish. A nonspecific control siRNA was used as negative control (Ctrl). Where required, empty pcDNA-HA vector was added to ensure equal total amounts of plasmid DNA in each transfection.

Design and screening of siRNAs. Complementary DNA sequences consisting of a 50-bp fragment of $\Delta$ Np63-WT and R304W mutant were cloned into pRL-TK (Renilla luciferase) and pGL-TK (Photinus luciferase) vectors, respectively. Site-directed mutagenesis primers were designed manually based on p63 sequence information. In order to screen all possible sequences containing the R304W mutation, 19 siRNAs were designed, each of which consisting of a 19-nucleotide sequence with two uracil nucleotide overhangs. Each siRNA molecule under evaluation was co-transfected into HEK-293E cells, seeded at 100000 cells per well in a 12-well plate, at a concentration of $40 \mathrm{nM}$ with either a wild-type p63 luciferase reporter construct ( $\mathrm{pRL}-\mathrm{TK}-\mathrm{WT}$ ) or a mutant p63 luciferase reporter construct (pGLTK-R304W). Luciferase activity was measured using the luciferase reporter assay $48 \mathrm{~h}$ after transfection. PSV- $\beta$ gal reporter vector was also co-transfected and $\beta$ galactosidase activity was used for transfection efficiency normalization. Not specific siRNA was used as a negative control.

Western blot analysis. HEK-293E cells were incubated for $48 \mathrm{~h}$ after transfection, then washed with PBS and lysed with Trypsin 1x. The extracted protein samples were denatured at $98^{\circ} \mathrm{C}$ for $10 \mathrm{~min}$ before being resolved on a SDS-10\% polyacrylamide gels at $10 \mu \mathrm{g}$ concentration. Gels were then transferred using polyvinylidene fluoride membranes. The expression of HA-tagged and Myc-tagged constructs was detected with an anti-HA monoclonal mouse antibody (Abcam, Cambridge, UK, 1:500 dilution) and an anti-Myc monoclonal mouse antibody (Celle Signalling Technology, Danvers, MA, USA, 1:500 dilution), respectively. An anti- $\beta$-actin antibody (Sigma, Saint Louis, MO, USA, 1:50000 dilution) was used as an endogenous control. Membranes were washed with PBSTween for $10 \mathrm{~min}$ three times before being stained with anti-mouse secondary antibody (Sigma, 1: 10000 dilution) for $1 \mathrm{~h}$ at room temperature. Detection was performed with ECL kit (Perkin Elmer, Waltham, MA, USA).

Real-time qPCR. Total RNA was extracted by the RNAeasy kit (Qiagen, Hilden, Germany) and quantified by spectrophotometric analysis. Total RNA (500ng) was used for reverse transcription using GoScript Reverse Transcription System kit (Promega, Fritchburg, WI, USA) following the manufacturer's instructions. Human $\beta$-actin mRNA was used as housekeeping gene for quantity normalization. Primer pairs used in $\mathrm{PCR}$ reactions are:

$\beta$-act forward 5'-CTGGCACCACACCTTCTACAATG- 3 ', $\beta$-act reverse $5^{\prime}$-TAGCAC AGCCTGGATAGCAAC-3'. K14 forward 5'-CGACCTGGAAGTGAAGATCCG-3', K14 reverse 5'-CACACTCATGCGCAGGTTCAA-3'. PERP forward 5'-TGTGGTGGAAA TGCTCCCAAG-3', reverse 5'-CCAATCACTCTCAGGAAGACAAGC-3'. EVPL forward 5'-TGCCCGAGCACAACATCCTG-3', reverse 5'-GACGCCGCCTTCAGTAGGTCTC-3'.

iPSC differentiation and transfection. Human corneal fibroblasts were isolated from cadaveric cornea as described previously ${ }^{24}$ and grown in DMEM (Invitrogen) 10\% FCS. Cell growth was arrested by mitomycin treatment $(8 \mu \mathrm{g} / \mathrm{ml}$; Sigma) for $3 \mathrm{~h}$ followed by washing with medium and incubation overnight. For the preparation of conditioned medium, growth medium was replaced by epithelial medium containing $60 \%$ DMEM+GlutMax, $30 \% \mathrm{~F} 12,10 \%$ fetal clone II, $5 \mu \mathrm{g} / \mathrm{ml}$ insulin, $0.5 \mu \mathrm{g} / \mathrm{ml}$ hydrocortisone, $10 \mathrm{ng} / \mathrm{ml}$ EGF, $0.2 \mathrm{mM}$ adenine and $10 \mathrm{mM}$ choleratoxin. Conditioned media were collected ( $20 \mathrm{ml}$ per T75 flask) every day for up to 10 days and stored at $-20^{\circ} \mathrm{C}$. 
a
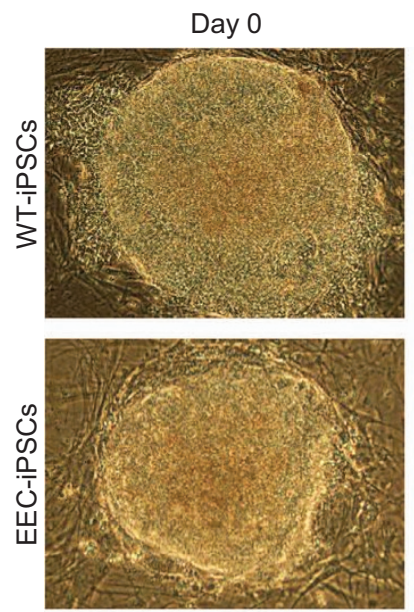

b

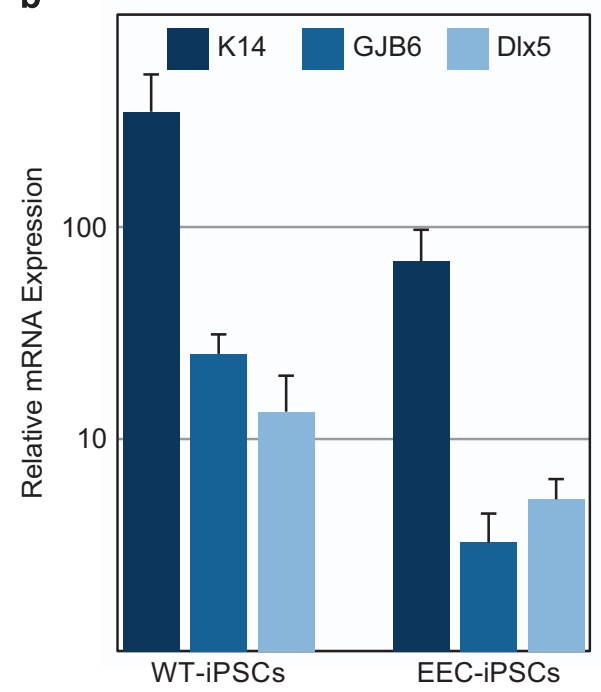

Day 10
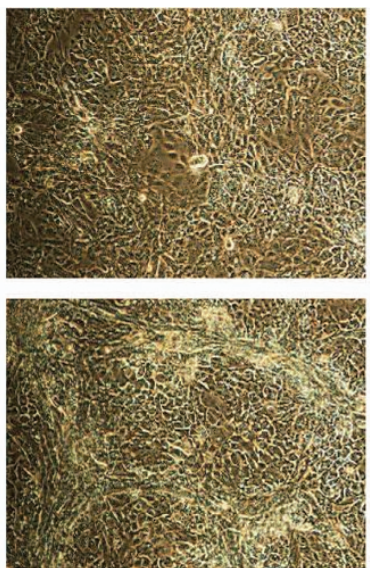

C

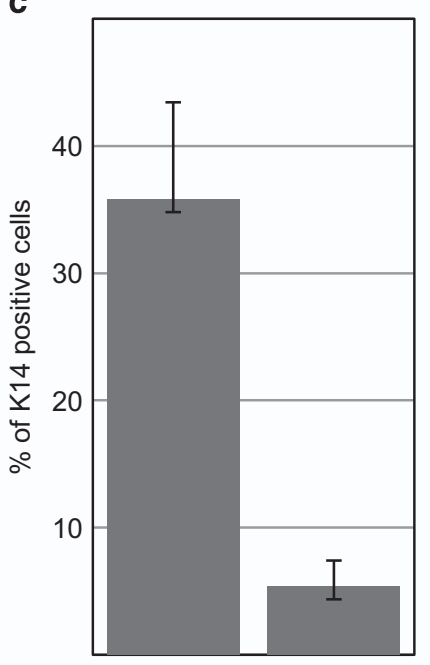

Day 13
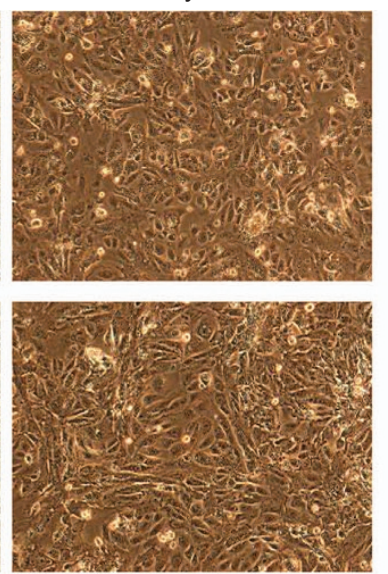

d
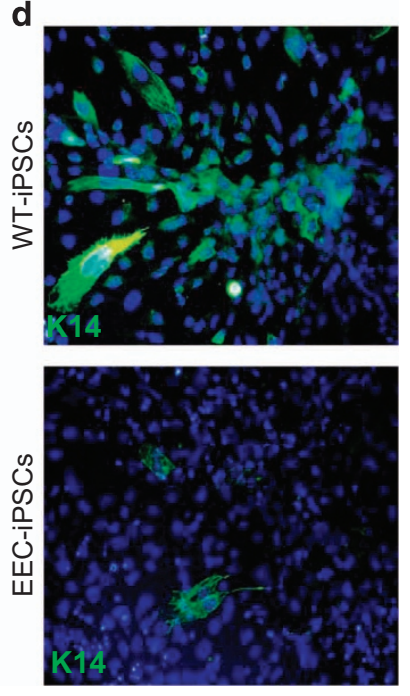

e

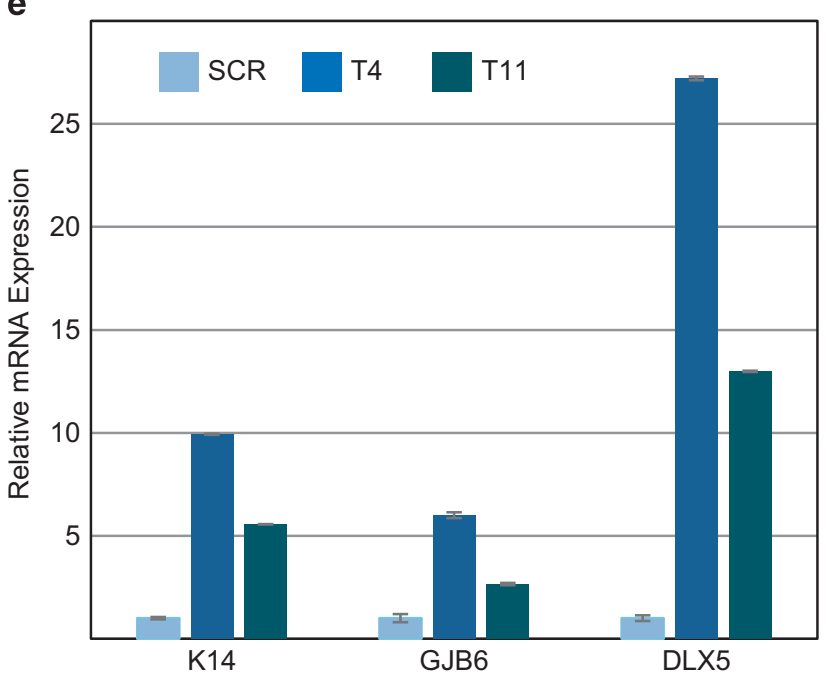

f

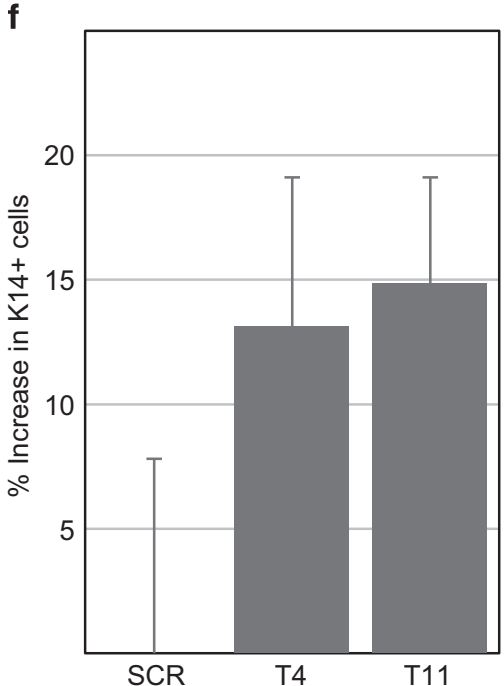

iPSC were grown and differentiated as described previously. ${ }^{7,24}$ In order to induce the differentiation of the iPSC lines into corneal epithelial cells, iPSC lines were seeded on collagen IV-coated dishes $(0.5 \mathrm{mg} / \mathrm{ml}$; Sigma) in the presence of conditioned media. IPSCs were transfected using Lipofectamine RNAiMAX
(Invitrogen) according to the manufacturer's instructions with nonspecific control siRNA (SCR) or mutated allele-specific siRNAs (T4, T11), on days 8-10 during differentiation at a concentration of $40 \mathrm{nM}$ and medium was replaced the next day. 
Figure 3 Rescue of corneal epithelial differentiation of EEC-iPSCs by mutated-p63 allele-specific silencing RNAs. WT-iPSCs and EEC-iPSCs were subjected to corneal epithelial differentiation protocol. Phase contrast pictures of WT-iPSCs and EEC-iPSCs at days 0,10 and 13 of differentiation are shown in a. Cells were harvested at days $13-15$ of differentiation and subjected to RT-qPCR analysis of the indicated genes (b), flow cytometry analysis (c) and immunofluorescence staining (d) of K14. EEC-iPSCs were transfected with nonspecific control silencing RNA (SCR) or specific siRNAs against p63 mutated allele (T4 and T11) at days 8-10 during differentiation into corneal epithelial cells. Cells were harvested at day 13-15 and subjected to RT-qPCR analysis of the indicated genes (e) and flow cytometry analysis of K14 (f). Gene expression was normalized to GAPDH housekeeping gene (b and $\mathbf{e}$ ) and data represent the fold change in gene expression compared with undifferentiated cells (b) or compared with SCR (e). Flow cytometry data represent the percentage of $\mathrm{K} 14+$ cells in the cell population (c) and the relative increase in the K14+ cells compared with SCR (f). Results are presented as mean \pm S.D. (Scale bar, $100 \mu \mathrm{m}$ )

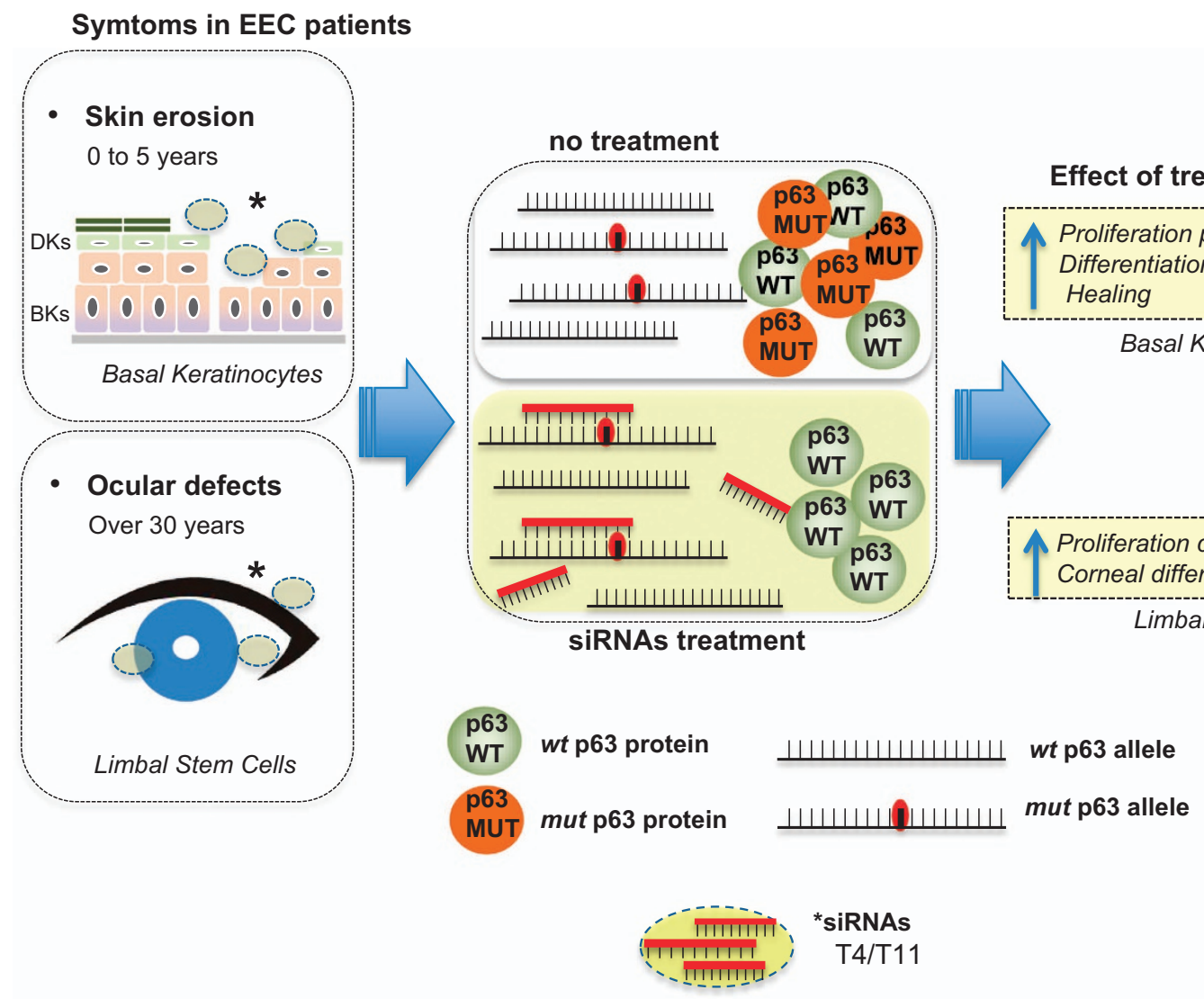

Figure 4 Schematic representation of siRNA-based therapeutic strategy in EEC disease. siRNAs identified could be used topically to cure/delay selected clinical onset, with high impact in the patient quality of life, such as skin erosion and ocular defects. Treatment with selected siRNAs (T4/T11 for p63-R304W) will silence the expression of p63 mutated allele, allowing p63-WT to transcriptionally activate target genes and to rescue proliferation and differentiation in basal keratinocytes (BK) and limbal stem cells (LSC)

Analysis of iPSC differentiation. Cells were harvested after 13-15 days of differentiation and subjected to the assays below. RNA was extracted using TRI Reagent (Sigma) and cDNA was synthesized from $2 \mu \mathrm{g}$ RNA using MultiScribe MuLV Reverse Transcription Kit (Applied Biosystems, Foster City, CA, USA). qRT-PCR was performed in triplicates using SYBR Green (Bioline, London, $\mathrm{UK}$ ) and specific primers (sequences available on request). Each reaction contained $15 \mu \mathrm{l}$ primers and SYBR-Green PCR Master Mix and $5 \mu \mathrm{l}$ cDNA. Human GAPDH mRNA was used as housekeeping gene for normalization. The relative expression of each transcript was calculated as a fold change relative to normalized control sample of differentiated iPSC transfected with SCR. Immunofluorescent staining of $\mathrm{K} 14$ and imaging as well as flow cytometry was performed as previously reported. ${ }^{7,24}$ Briefly, cells were fixed with acetone for $10 \mathrm{~min}$ at $-20^{\circ} \mathrm{C}$. After washing with PBS, cells were incubated with $0.5 \% \mathrm{BSA}, 0.5 \%$ saponin and donkey serum for $30 \mathrm{~min}$. Primary antibody for K14 (Millipore, Billerica, MA, USA) was added for $45 \mathrm{~min}$ at room temperature. Acquisition was performed on FACS-CyAn using CellQuest software (BD Biosciences, Franklin Lakes, NJ, USA).

\section{Conflict of Interest}

The authors declare no conflict of interest.

Acknowledgements. We thank Drs. Nishino I and Noguchi $S$ for kindly providing us with luciferase reporter plasmids (phRL-TK-Renilla; pGL3-TK-Photinus). RS-F has received funding from the European Union's - Seventh Framework Programme (FP7/2007-2013]) under grant agreement no. 618432 - MC - Epi-PathoStem, and by the Ministry of Science, Technology, and Space, Israel and the Ministere de L'Education National de L'Enseignement Supérieur de la Recherche (3-11985).

1. Rinne T, Brunner HG, van Bokhoven H. p63-associated disorders. Cell Cycle 2007; 6 : 262-268.

2. Di lorio E, Kaye SB, Ponzin D, Barbaro V, Ferrari S, Böhm E et al. Limbal stem cell deficiency and ocular phenotype in ectrodactyly-ectodermal dysplasia-clefting syndrome caused by p63 mutations. Ophthalmology 2012; 119: 74-83.

3. Clements SE, Techanukul T, Coman D, Mellerio JE, McGrath JA. Molecular basis of EEC (ectrodactyly, ectodermal dysplasia, clefting) syndrome: five new mutations in the 
DNA-binding domain of the TP63 gene and genotype-phenotype correlation. $\mathrm{Br} J$ Dermatol 2010; 162: 201-207.

4. Celik TH, Buyukcam A, Simsek-Kiper PO, Utine GE, Ersoy-Evans S, Korkmaz A et al. A newborn with overlapping features of AEC and EEC syndromes. Am J Med Genet 2011; 155A: 3100-3103.

5. Rinne T, Hamel B, van Bokhoven $H$, Brunner HG. Pattern of p63 mutations and their phenotypes-update. Am J Med Genet 2006; 140: 1396-1406.

6. Shalom-Feuerstein R, Lena AM, Zhou H, De La Forest Divonne S, Van Bokhoven H, Candi E et al. $\triangle \mathrm{Np63}$ is an ectodermal gatekeeper of epidermal morphogenesis. Cell Death Differ 2011; 18: 887-896.

7. Shalom-Feuerstein R, Serror L, Aberdam E, Müller FJ, van Bokhoven H, Wiman KG et al. Impaired epithelial differentiation of induced pluripotent stem cells from ectoderma dysplasia-related patients is rescued by the small compound APR-246/PRIMA-1MET. Proc Natl Acad Sci USA 2013; 110: 2152-2156.

8. Felipe AF, Abazari A, Hammersmith KM, Rapuano CJ, Nagra PK, Peiro BM. Corneal changes in ectrodactyly-ectodermal dysplasia-cleft lip and palate syndrome: case series and literature review. Int Ophthalmol 2012; 32: 475-480.

9. Celli J, Duijf P, Hamel BC, Bamshad M, Kramer B, Smits AP et al. Heterozygous germline mutations in the p53 homolog p63 are the cause of EEC syndrome. Cell 1999; 99: 143-153.

10. van Bokhoven H, Hamel BC, Bamshad M, Sangiorgi E, Gurrieri F, Duijf PH et al. p63 Gene mutations in eec syndrome, limb-mammary syndrome, and isolated split hand-split foot malformation suggest a genotype-phenotype correlation. Am J Hum Genet 2001; 69: 481-492.

11. Rivetti di Val, Cervo P, Lena AM, Nicoloso M, Rossi S, Mancini M et al. p63-microRNA feedback in keratinocyte senescence. Proc Natl Acad Sci USA 2012; 109: 1133-1138.

12. Candi E, Cipollone R, Rivetti di Val Cervo P, Gonfloni S, Melino G, Knight R. p63 in epithelia development. Cell Mol Life Sci 2008; 65: 3126-3133.

13. Candi E, Rufini A, Terrinoni A, Giamboi-Miraglia A, Lena AM, Mantovani R et al. DeltaNp63 regulates thymic development through enhanced expression of FgfR2 and Jag2. Proc Natl Acad Sci USA 2007; 104: 11999-12004.

14. Viganò MA, Lamartine J, Testoni B, Merico D, Alotto D, Castagnoli C et al. New p63 targets in keratinocytes identified by a genome-wide approach. EMBO J 2006; 25: 5105-5116.

15. Romano RA, Smalley K, Magraw C, Serna VA, Kurita T, Raghavan S et al. $\Delta$ Np63 knockout mice reveal its indispensable role as a master regulator of epithelial development and differentiation. Development 2012; 139: 772-782.

16. Latina A, Viticchiè G, Lena AM, Piro MC, Annicchiarico-Petruzzelli M, Melino G et al. $\Delta$ Np63 targets cytoglobin to inhibit oxidative stress-induced apoptosis in keratinocytes and lung cancer. Oncogene 2015 (doi:10.1038/onc.2015.222; e-pub ahead of print).

17. Viticchiè G, Agostini M, Lena AM, Mancini M, Zhou H, Zolla L et al. p63 supports aerobic respiration through hexokinase II. Proc Natl Acad Sci USA 2015; 112: 11577-11582.

18. Carroll DK, Carroll JS, Leong CO, Cheng F, Brown M, Mills AA et al. p63 regulates an adhesion programme and cell survival in epithelial cells. Nat Cell Biol 2006; 8: 551-561.

19. Candi E, Rufini A, Terrinoni A, Dinsdale D, Ranalli M, Paradisi A et al. Differential roles of p63 isoforms in epidermal development: selective genetic complementation in p63 null mice. Cell Death Differ 2006; 13: 1037-1047.

20. Rufini A, Weil M, McKeon F, Barlattani A, Melino G, Candi E. p63 protein is essential for the embryonic development of vibrissae and teeth. Biochem Biophys Res Commun 2006; 340: 737-741.

21. Ihrie RA, Marques MR, Nguyen BT, Horner JS, Papazoglu C, Bronson RT et al. Perp is a p63-regulated gene essential for epithelial integrity. Cell 2005; 120: 843-856.
22. Browne G, Cipollone R, Lena AM, Serra V, Zhou H, van Bokhoven H et al. Differential altered stability and transcriptional activity of $\Delta \mathrm{Np63}$ mutants in distinct ectodermal dysplasias. J Cell Sci 2011; 124: 2200-2207.

23. Noguchi S, Ogawa M, Kawahara G, Malicdan MC, Nishino I. Allele-specific gene silencing of mutant mRNA restores cellular function in ullrich congenital muscular dystrophy fibroblasts. Mol Ther Nucleic Acids 2014; 3: e171.

24. Shalom-Feuerstein R, Serror L, De La Forest Divonne S, Petit I, Aberdam E, Camargo L et al. Pluripotent stem cell model reveals essential roles for miR-450b-5p and miR-184 in embryonic corneal lineage specification. Stem Cells 2012; 30: 898-909.

25. Melo SP, Lisowski L, Bashkirova E, Zhen HH, Chu K, Keene DR et al. Somatic correction of junctional epidermolysis bullosa by a highly recombinogenic AAV variant. Mol Ther 2014; 22: 725-733.

26. Keswani SG, Balaji S, Le L, Leung A, Lim FY, Habli M et al. Pseudotyped adeno-associated viral vector tropism and transduction efficiencies in murine wound healing. Wound Repair Regen 2012; 20: 592-600.

27. Sebastiano V, Zhen HH, Haddad B, Bashkirova E, Melo SP, Wang P et al. Human COL7A1corrected induced pluripotent stem cells for the treatment of recessive dystrophic epidermolysis bullosa. Sci Transl Med 2014; 6: 264ra163.

28. Atkinson SD, McGilligan VE, Liao H, Szeverenyi I, Smith FJ, Moore CB et al. Development of allele-specific therapeutic siRNA for keratin 5 mutations in epidermolysis bullosa simplex. $J$ Invest Dermatol 2011; 131: 2079-2086.

29. Leslie Pedrioli DM, Fu DJ, Gonzalez-Gonzalez E, Contag CH, Kaspar RL, Smith FJ et al. Generic and personalized RNAi-based therapeutics for a dominant-negative epidermal fragility disorder. J Invest Dermatol 2012; 132: 1627-1635.

30. Liao H, Irvine AD, Macewen CJ, Weed KH, Porter L, Corden LD et al. Development of allelespecific therapeutic siRNA in Meesmann epithelial corneal dystrophy. PLoS One 2011; 6: e28582.

31. Allen EH, Atkinson SD, Liao H, Moore JE, Leslie Pedrioli DM et al. Allele-specific siRNA silencing for the common keratin 12 founder mutation in Meesmann epithelial corneal dystrophy. Invest Ophthalmol Vis Sci 2013; 54: 494-502.

32. Courtney DG, Atkinson SD, Allen EH, Moore JE, Walsh CP, Pedrioli DM et al. siRNA silencing of the mutant keratin 12 allele in corneal limbal epithelial cells grown from patients with Meesmann's epithelial corneal dystrophy. Invest. Ophthalmol Vis Sci 2014a; 55: 3352-3360.

33. Courtney DG, Atkinson SD, Moore JE, Maurizi E, Serafini C, Pellegrini G et al. Development of allele-specific gene-silencing siRNAs for TGFBI Arg124Cys in lattice corneal dystrophy type I. Invest Ophthalmol Vis Sci 2014b; 55: 977-985.

(i) Cell Death and Disease is an open-access journal published by Nature Publishing Group. This work is licensed under a Creative Commons Attribution 4.0 International License. The images or other third party material in this article are included in the article's Creative Commons license, unless indicated otherwise in the credit line; if the material is not included under the Creative Commons license, users will need to obtain permission from the license holder to reproduce the material. To view a copy of this license, visit http://creativecommons.org/licenses/by/4.0/ 